\title{
Partially-indirect Bimanual Input with Gaze, Pen, and Touch for Pan, Zoom, and Ink Interaction
}

\author{
Ken Pfeuffer, Jason Alexander, Hans Gellersen \\ Lancaster University \\ Lancaster, United Kingdom \\ \{k.pfeuffer, j.alexander, h.gellersen\}@lancaster.ac.uk
}

\begin{abstract}
Bimanual pen and touch UIs are mainly based on the direct manipulation paradigm. Alternatively we propose partiallyindirect bimanual input, where direct pen input is used with the dominant hand, and indirect-touch input with the nondominant hand. As direct and indirect inputs do not overlap, users can interact in the same space without interference. We investigate two indirect-touch techniques combined with direct pen input: the first redirects touches to the user's gaze position, and the second redirects touches to the pen position. In this paper, we present an empirical user study where we compare both partially-indirect techniques to direct pen and touch input in bimanual pan, zoom, and ink tasks. Our experimental results show that users are comparatively fast with the indirect techniques, but more accurate as users can dynamically change the zoom-target during indirect zoom gestures. Further our studies reveal that direct and indirect zoom gestures have distinct characteristics regarding spatial use, gestural use, and bimanual parallelism.
\end{abstract}

\section{ACM Classification Keywords}

H.5.2. Information interfaces and presentation: User Interfaces: Input devices and strategies

\section{Author Keywords}

Bimanual input; pen and touch; gaze; pan and zoom; direct and indirect input.

\section{INTRODUCTION}

Direct pen and touch manipulation is increasingly supported on tablet and large display computers. This efficiently enables asymmetric bimanual input with the pen in the dominant hand and multi-touch of the non-dominant hand [6, 8, 12]. For instance, pan and zoom gestures for UI navigation together with a pen for precise inking is useful for sketching $[12,30]$, text editing $[10,32]$, or vector graphics work $[6,11,31]$.

In this context, we investigate how to partially integrate indirect input to bimanual pen and touch UIs. We propose to

Permission to make digital or hard copies of all or part of this work for personal or classroom use is granted without fee provided that copies are not made or distributed for profit or commercial advantage and that copies bear this notice and the full citation on the first page. Copyrights for components of this work owned by others than ACM must be honored. Abstracting with credit is permitted. To copy otherwise, or republish, to post on servers or to redistribute to lists, requires prior specific permission and/or a fee. Request permissions from Permissions@ acm.org.

CHI'16, May 07-12, 2016, San Jose, CA, USA

(C) 2016 ACM. ISBN 978-1-4503-3362-7/16/05 ..\$15.00

DOI: http://dx.doi.org/10.1145/2858036.2858201

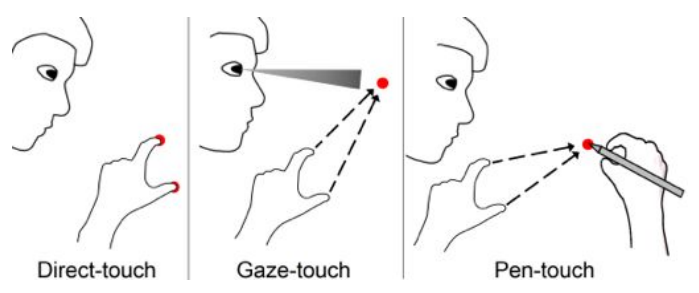

Figure 1: We investigate the indirect gaze-touch and pen-touch techniques in comparison to direct-touch for use in bimanual interfaces.

use the dominant hand for standard direct pen input, while the non-dominant hand performs indirect-touch input. With indirection, users gain remote, occlusion-free, and precise input $[4,20,29]$. As direct and indirect inputs do not physically overlap, users can employ two-handed input in the same space without interference.

We explore this partially-indirect bimanual input with the following two indirect-touch techniques. These are combinations of gaze, pen, and touch input, and by design can be utilised side by side with direct pen input (Fig. 1):

Pen-touch: On a pen and touch display, the user's work is often centered around the pen that is held in the dominant hand. Pen-touch is designed for these cases, as touch input is automatically redirected to the pen position. Users point the pen at the target, and perform indirect-touch gestures from any close or remote position.

Gaze-touch: Most user interactions naturally happen in the area of the user's visual attention. Gaze-touch utilises this by redirecting touch input to the user's gaze position. Users look at a target, and perform indirect-touch from remote; a technique that has shown high potential for interactive surfaces $[20,24,25]$.

In a bimanual pen and touch experiment, we compare these two indirect techniques to default direct pen and touch interaction. In this experiment, users navigate the canvas with pan and zoom by touch, while the pen is used to select and draw objects. Two variations of this task are tested: one where users alternate between pen and touch, and the other one where they use both in parallel.

Study results show the partially-indirect bimanual configuration has (1) comparable time performance to direct manipulation while (2) it improves in accuracy of zooming. Further post-hoc analysis of gestural and spatial characteristics 
showed that (3) users perform zoom gestures faster and more frequent with indirect-touch, (4) indirect-touch can lead to more (pen-touch) or less (gaze-touch) bimanual parallelism than direct touch, and (5) users keep pen and touch modalities spatially further apart with indirect-touch.

Our contributions are (1) the concept and techniques that use the pen with the dominant hand and indirect-touch with the non-dominant hand, (2) a bimanual pen and touch experiment comparing two indirect-touch techniques to direct touch, and (3) novel findings about pinch-to-zoom accuracy, visual behaviour, bimanual parallelism, and direct vs indirect input.

\section{RELATED WORK}

\section{Bimanual Pen and Touch Interaction}

The efficiency of bimanual input depends on the task, e.g. it can be beneficial to use two hands over one in image alignment, geometry manipulation, or multi-target interaction [7, $13,14,15]$. These tasks involve control of multiple degrees of freedom or multiple sub-tasks, which are distributed over both hands. Concurrent manipulation with two hands can increase user performance, but also a logical structure across the hands is relevant for efficient bimanual interactions [7, $8,16]$. Pen and touch is such a logical division of labour, where users can perform main inking tasks with the dominant hand, and supportive multi-touch manipulation with the non-dominant hand $[6,12]$. The alternating or simultaneous use of the two modalities can provide new opportunities for interaction $[6,10,12,32,33]$.

Researchers studied direct pen and touch interaction in comparisons to different configurations of the two modalities. Brandl et al. compared pen/touch against pen/pen and touch/touch configurations. Their study indicated pen/touch to be superior for a task based on drawing with the pen, while performing pan and zoom gestures with touch [6]. Lopes et al. compared pen and touch to pen only configurations. Touch based navigation with pen based sketching was found superior in 3D manipulation tasks [17]. Matulic and Norrie investigated configurations where a pen is combined with various supportive direct-touch techniques [18]. In a task that involves both sequential/simultaneous use (trace a polyline with pen and change pen mode with touch), the results included that a maintained touch posture can increase the user's ability for bimanual coordination with the pen. Our research complement these studies with an additional comparison of direct pen and touch to new bimanual configurations combining direct pen with indirect-touch.

\section{Indirect-touch and Gaze Interaction}

Gaze has been recently explored for indirect pen and touch interactions on a direct display by Pfeuffer et al. [20, 21]. They introduced gaze-touch [20], a technique based on the division of labour 'gaze selects, touch manipulates', and interaction opportunities when a direct modality is used indirectly. Their work on gaze-shifting involved pen and touch modalities [21], focusing on switching a direct input device between direct and indirect input by gaze. Our work shares the use of gaze-touch and the investigation of direct/indirect input, but we focus on combined direct and indirect input with two hands, and further we evaluate this approach in a bimanual pen and touch experiment.

A range of works investigated gaze based indirect-touch for desktop and remote display setups [20, 24, 25, 26]. Stellmach and Dachselt investigated gaze based pan and zoom for map navigation [23], and user feedback indicated preference to a gaze-based zooming approach with touch. Pfeuffer et al. compared direct-touch to gaze based indirect-touch on a remote screen. they found increased accuracy for the gaze condition, and account it to the avoidance of the fat-finger problem [22]. On large projections, Turner et al. compared touch based translation to various configurations where gaze supports translation; indicating that the addition of gaze can improve dragging performance [25].

Advantages of indirect-touch in general were studied priorly to alleviate issues associated with direct input, such as occlusion [27, 28], precision [4, 29], or remote interaction [1, 5]. Proposed indirect-touch techniques range from simple offsets of single-touch [28] to more complex bimanual touch selection techniques [4] and dedicated gestures/widgets for proxies to remote targets $[1,5,29]$. Although these methods enable indirect-touch, they involve additional steps such as proxy generation before users can actually manipulate the target, which we think hampers a dynamic interplay with direct pen input. With gaze-touch and pen-touch, we investigate techniques where touches immediately redirect to the target.

\section{INTERACTION TECHNIQUES}

We first describe the investigated interaction techniques and then analyse their interaction properties:

DT: Direct-touch: This technique is standard on pen and touch interfaces, where users touch the position they want to manipulate, and the action begins immediately at touch down. Current pen and touch displays employ this technique for multi-touch input by the user, that is combined with the inking mode of the pen.

GT: Gaze-touch (Figure 2): In graphical context such as pen and touch displays, the user's visual attention is often correlated with the actual area that users interact in. Researchers have thus suggested to redirect the effect of touch gestures toward the user's gaze position on the screen [24, 25]. This provides benefits such as whole-surface reachability, occlusionfree, and precise input through indirect-touch (more details in [20]). Essentially, gaze-touch consists of a two step interaction: users look at a target to select it, and then touch down and perform a gesture to indirectly manipulate it.

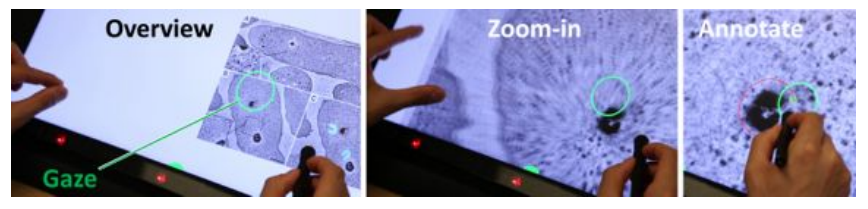

Figure 2: Gaze-touch: from an overview medical image, users quickly zoom into their gaze position to then use the pen for annotations. The user's gaze position is indicated with the green circle. 
PT: Pen-touch (Figure 3): Within a pen and touch interaction context, the user's focus of interaction is often already located around the pen that is held in the dominant hand. For example, a user draws in a graphical model with the pen, and then drags the same model with touch. Pen-touch is based on this premise as a new technique where the effect of touch gestures is redirected to the pen's position. This allows users to perform touch gestures on a target that is already occupied by the pen, and focuses the user's interaction around the pen device. The touch redirection works during pen down and hover events.

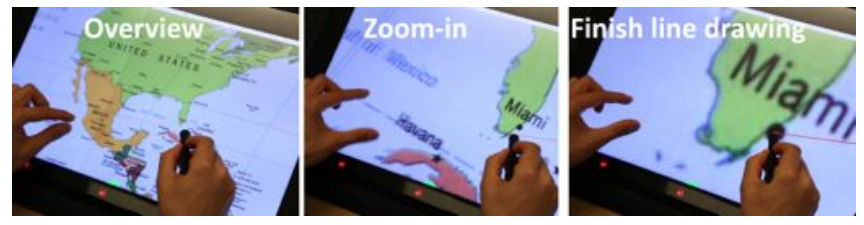

Figure 3: Pen-touch: While users are drawing a line, users can zoom into the pen's position and then precisely finish the line drawing.

\section{Analysis of Interaction Properties}

We now analyse the three techniques with a focus on bimanual interaction properties. We extend Pfeuffer et al.'s [20] comparison of direct-touch vs. gaze-touch with a focus on bimanual pen and touch interaction. The interaction properties are summarised in table 1. Notably, all techniques still support concurrent pan and zoom with two-touch gestural input.

\begin{tabular}{l|l|l|l} 
& DT & GT & PT \\
\hline Gesture target & Touch & Gaze & Pen \\
\hline Hands needed & 1 hand & 1 hand & 2 hands \\
\hline No occlusion & - & $\checkmark$ & $\checkmark$ \\
\hline No interference & - & $\checkmark$ & $\checkmark$ \\
\hline Same-target Simultaneity & - & $\checkmark$ & $\checkmark$ \\
\hline Separate-target Simultaneity & $\checkmark$ & $\checkmark$ & - \\
\hline Dynamic targeting & - & $\checkmark$ & $\checkmark$
\end{tabular}

Table 1: Summary of differences between techniques

Division of Labour (Table 2): In general, all techniques follow Hinckley et al's division of labour between modalities pen writes, touch manipulates [12]. A further division of labour occurs for the touch manipulates part, that has distinct implications on the interaction with the technique.

Direct-touch frees the user's gaze and pen input during touch gestural interactions, but requires moving their hands to where they want to perform the gesture. For instance, it can be appropriate when users want to clearly indicate where they touch to collaborators. Gaze-touch does not require relocating either pen or touch to issue gestures, but requires the user to explicitly direct their gaze to a target. Thus it is appropriate for interactions where the hand needs to keep out of the user's view. Pen-touch does not use gaze explicitly nor are users required to move the touch-hand to the gesture target, but requires the user to move the pen to the gesture target. This essentially segments touch gesture selection and manipulation based on Guiard's proposition that the dominant hand performs precise, and the non-dominant hand performs coarse tasks [8]. For example, the technique is appropriate when

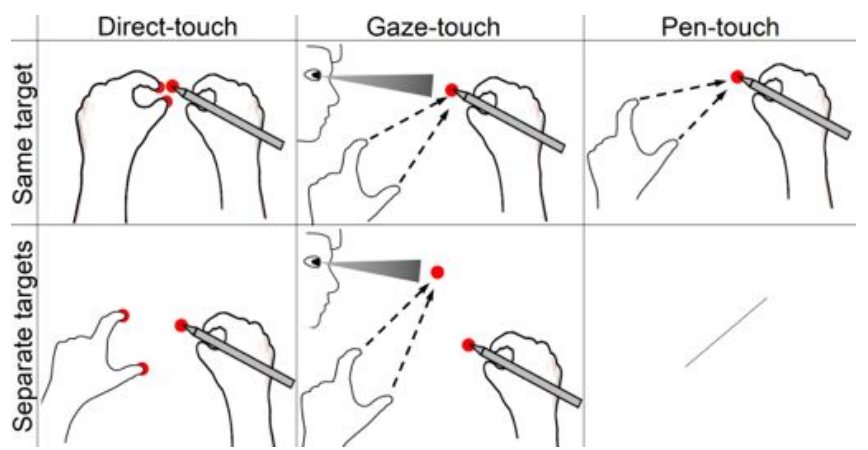

Figure 4: Simultaneity: each techniques has different feasibility for simultaneous interaction on the same or on separate targets.

precise target selection with the pen tip is required, such as CAD modelling.

\begin{tabular}{l|l|l|l} 
& \multicolumn{2}{|l|}{$\begin{array}{l}\text { Touch manipulates } \\
\text { (non-dominant hand) }\end{array}$} & Pen inks \\
& Select & Manipulate & \\
\hline Direct-touch & Touch & Touch & Pen \\
\hline Gaze-touch & Gaze & Touch & Pen \\
\hline Pen-touch & Pen & Touch & Pen
\end{tabular}

Table 2: The techniques share the overall division of labour, and vary for the 'select' sub-task during touch gestures. The 'manipualate' part is touch only across all techniques to support all standard touch gestures.

Occlusion: Direct-touch naturally induces occlusion when the user's hand/arm is on the screen [27], which increases with two-handed input. Both indirect techniques (gaze-touch / pen-touch) are occlusion-free as the hand is decoupled from the manipulation, only the hand that holds the pen can still cast occlusion.

Interference: Direct-touch is prone to interference: when users want to interact with one target with both hands, one hand spatially interferes with the other hand, which requires alternating use of pen and touch modalities. Both indirect techniques enable same-target manipulation with both modalities.

Same-target Simultaneity (Figure 4 top): Same target interaction occurs when users perform two modes simultaneously on one target such as drawing a curve while adjusting its roundness. This works with gaze-touch and pen-touch as users can directly ink with the pen, and at the same time indirectly manipulate the same target. At touch down users look at it (gaze-touch), while for pen-touch the target is already at the pen's position. With direct-touch, users cannot exactly manipulate the same target because of interfering hands, except if the target area is large enough to be manipulated from multiple points.

Separate-targets Simultaneity (Figure 4 bottom): Users interact with two separate targets simultaneously when for instance dragging an image while opening a folder with the other hand. This works for direct-touch and gaze-touch: users can select a point with the dominant hand (pen), and simultaneously select a different point by touching on it (directtouch) or looking at a different target (gaze-touch). This does 


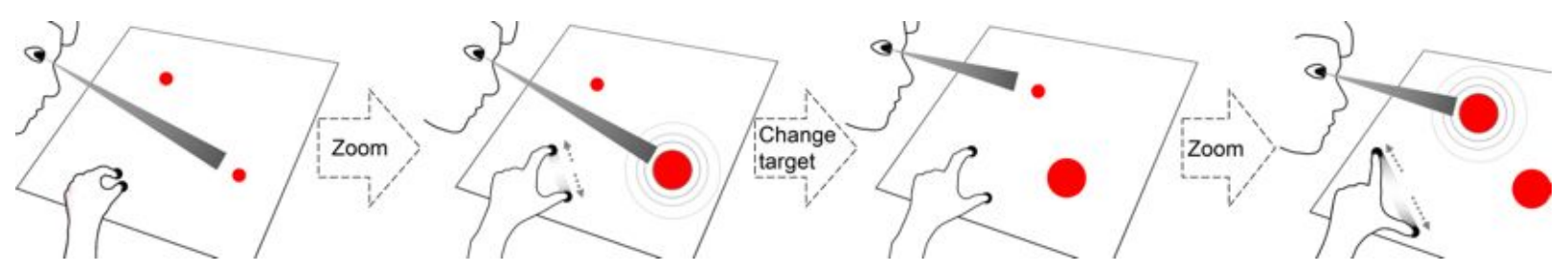

Figure 5: Dynamic Targeting: With indirect touch techniques such as gaze-touch, users can change the target during the gesture without lifting fingers.

not work with pen-touch, as any touch is redirected to the pen's position, and the system would have to choose between using either pen only or pen-touch input.

Dynamic targeting (Figure 5): the established direct-touch paradigm resembles real-world physics, and when users 'grab' an object, the touch positions are glued to the object's local position that users initially touched. To interact with another target, users lift their fingers and move them to the new target.

This is different from the indirect techniques (gaze-touch, pen-touch) where users can dynamically change the target during a touch gesture. Without lifting their fingers, users can move the pen or their gaze to a different target. For instance, when performing pinch-to-zoom, users can adjust their zooming pivot while they zoom-in to achieve more precise navigation. Thus Dynamic Targeting can increase the accuracy of touch manipulation. More accuracy can in turn lead to a decrease of the amount of panning and clutching operations that users perform during navigation [2].

\section{BIMANUAL PEN AND TOUCH EXPERIMENT}

To understand how the techniques compare in practice, we evaluate the performance of the three techniques in two tasks: one where pen and touch are used in alternation, and the other one where the modalities are used in parallel.

\section{Research Questions}

Task Completion Time: How does each technique affect the user's temporal performance in a sequential and simultaneous pen and touch task? The techniques have distinct properties for use in alternating and simultaneous use of pen and touch.

Accuracy: How does the Dynamic Targeting feature of indirect techniques come into play? For this we measure the accuracy of zoom gestures (the disparity between positions users zoom in vs. the actual target where users need to zoom).

Gesture Characteristics: Does the indirection through pentouch and gaze-touch affect the users gestures? Across the techniques, users perform the same type of gestures, only the target of the gesture varies with technique.

Parallelism between Pen and Touch: Does a technique involve more parallelism between the pen and touch modalities than others? Parallelism can be, but is not necessarily correlated with the efficiency of bimanual interaction $[7,16]$.

Spatial Distribution of Input Modalities: How do users couple the pen and touch modalities? Users touch at the manipu- lation point with direct-touch, but it is unclear whether users return to these patterns with the indirect techniques.

User Feedback: Do users like the familiar direct manipulation paradigm or come to prefer a new technique?

\section{Tasks}

We chose touch based pan and zoom with pen based drawing as the underlying task environment, a combination where users benefit from bimanual pen and touch inputs $[6,8,12]$. We use two tasks, one more suitable for sequential interaction and one more suitable for simultaneous interaction with the two modalities.

Sequence task (Figure 6a): In this task, users navigate to, and then select three targets. Users first zoom out to get an overview, and then zoom into the target area. When users find the actual target dots, they draw a circle around them to finish the task.

Parallel task (Figure 6b): In this task, users draw a line while navigating the canvas. Users first select the start point of the line, and then navigate toward the end point. The end point is not visible at the start, and therefore users zoom out to get an overview, and then zoom into the target area. During the navigation, the pen remains pressed on the screen. When the target is visible, users move the pen to the target, and lift up to finish.

Both tasks adapt Guiard et al's multiscale pointing task [9] for the part where users perform pan and zoom, similar to Nancel et al.'s investigation of pan and zoom techniques [19]. Participants navigate through an abstract interface with two groups of concentric circles (start and target group). The gray start group is where users begin the task and zoom out (Fig. 6, first two columns). When zoomed out enough, the orange target group becomes visible (6a-3). Users then zoom into the target group. The center of the target group is specifically offset from the center of the start group (Table 3). The angle between both circle groups is randomized for each trial. The last circle of the orange target group contains 10 gray dots that are randomly placed within it (6a-5). The zoom-in sub-part is finished when the initial zooming level is reached again, and the last circle of the orange target group is within the display's region (the last circle width $=450 \mathrm{px}$, all dots' width $=50 \mathrm{px}$ ).

The end of the pen task then becomes visible: the relevant dots are highlighted red (three dots for the sequence task, one dot for the parallel task, see Fig. $6 a-5$ and $6 b-5$ ). The target dots are randomly selected. For the sequence task, the first dot is randomly selected, and then the two closest neighbor dots are additionally selected as target dots. 


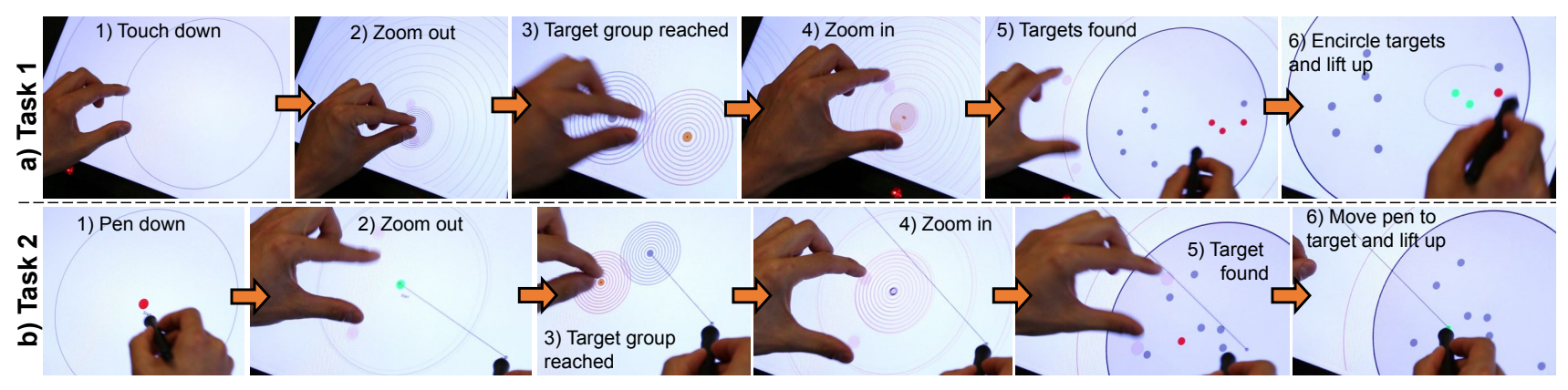

Figure 6: Substeps of the two study tasks on the example of the direct-touch technique

The sequence task finishes when the user has encircled all three dots (Fig. 6a-6), and if not, users can draw additional lines (but need not encircle all three again, only the remaining dots). Each dot is highlighted green when inside of a user's drawn lines.

For the parallel task, the task begins with users placing the pen at a centered dot before performing the pan \& zoom navigation (Fig. 6b-2). The task finishes when the pen moved within the ending dot's area (where it gets highlighted green, Fig. 6b-6)), and lifted up. If users lift the pen without being in the dot's area, the task is voided and will be repeated.

\section{Design and Factors}

Our experiment used a within subjects design. The task order was counterbalanced for each user, and the technique order was counterbalanced for each user using a balanced latin square. For both tasks, we used the same three distances (Table 3). The distance is the length that users navigate from start to end point of the pan and zoom task. The minimum distance was chosen as the minimum index of difficulty where pan and zoom becomes beneficial (ID=8, [9]). The remaining distances are steps of 3 indices of difficulty (using formula $\log _{2}(D / W+1)$ with fixed $\left.W=50 p x\right)$. Each distance was repeated 15 times. Within each task $\times$ technique block, users performed 45 trials $(=15 \times 3$ distances). The order of the distances was randomised within the block. Overall, this resulted in 2 tasks $\times 3$ techniques $\times 3$ distances $\times 15$ repetitions $=270$ trials per participant.

\begin{tabular}{l|l|l|l} 
& Small & Medium & Large \\
\hline ID & 8 & 11 & 14 \\
\hline Centimeter & 315 & 2532 & 20265 \\
\hline Pixel & 12751 & 102351 & 819151
\end{tabular}

Table 3: Study distance factors (for both tasks) in three metrics.

\section{Participants}

18 paid participants took part in the study. On average they were 26.7 years old ( $\mathrm{SD}=6.4,6$ female), and students or employees of the local university with mixed background. Only one user was left-handed, and we mirrored positional data post-hoc for a right-handers dataset. 5 users wore glasses, and 4 contact lenses. On a 1 (no experience) to 5 (expert) scale, users rated themselves as experienced with multi-touch (3.9, $\mathrm{SD}=1.1)$, and less experienced with eye gaze interaction $(2.6, \mathrm{SD}=1.38)$ and stylus interaction $(2.6, \mathrm{SD}=1.42)$.

\section{Procedure}

At first, users filled out a demographic questionnaire and conducted the gaze calibration. Then users performed the six task $\times$ technique blocks. Before each block, users performed up to five trials to get used to the technique and were instructed to be as fast as possible. After each block, users filled out a questionnaire with 6 Likert scale questions: 'The task with this technique was [easy to use | fast | precise | easy to learn | eye fatiguing | physically fatiguing (hand, arm, shoulder, or neck)']. Lastly, users filled out a ranking questionnaire and discussed why they preferred which technique. Overall, the study lasted 60-90 minutes.

\section{Apparatus}

We use a Wacom Cintiq 22HD pen and touch display with an Tobii EyeX eye tracker (30hz, Figure 7). The tracker is placed at the bottom border of the display. The display is oriented at a $45^{\circ}$ angle to enable comfortable reach, allows 10 -finger multi-touch at $120 \mathrm{hz}$, and has a Wacom Grip Pen. The user sits in front of the system with approximately $60 \mathrm{~cm}$ between the user's eyes and the eye tracker. Users were calibrated to the tracker at the beginning of the study using the standard EyeX application. We also conducted a 16-point accuracy test after each study session. The average accuracy was $1.51^{\circ}$ $\left(\mathrm{SD}=.58^{\circ}\right)$. The software is implemented in Java and runs on a 64-bit, 16GBRAM, quadcore i7@2.4GHz laptop. Simultaneous pen (WACOM Pen) and touch is detected with the Wacom SDK. Accidental touches that can occur from the pen holding hand are ignored by removing all touches that occur to the right of the pen tip (for right-handers).

The user's gaze was smoothed during the gaze-touch technique. As smoothing inherently introduces interaction delay, we use a more dynamic method: when users quickly moved their gaze (above $1050 \mathrm{px} / \mathrm{s}$ or $24^{\circ} / \mathrm{s}$ of visual angle), raw gaze data was used. Otherwise gaze data was averaged for $500 \mathrm{~ms}$ (includes 15 gaze samples), which helps to stabilise the jittery gaze cursor during fixations.

When users occluded the eye tracker (e.g. with a hand) or moved their head out of range, an error message was displayed to indicate the user to correct their position. This was explained and tried before study to avoid confusion. We considered gaze data as outliers when the eye tracker reported error (usually when users are out of range or blink). 


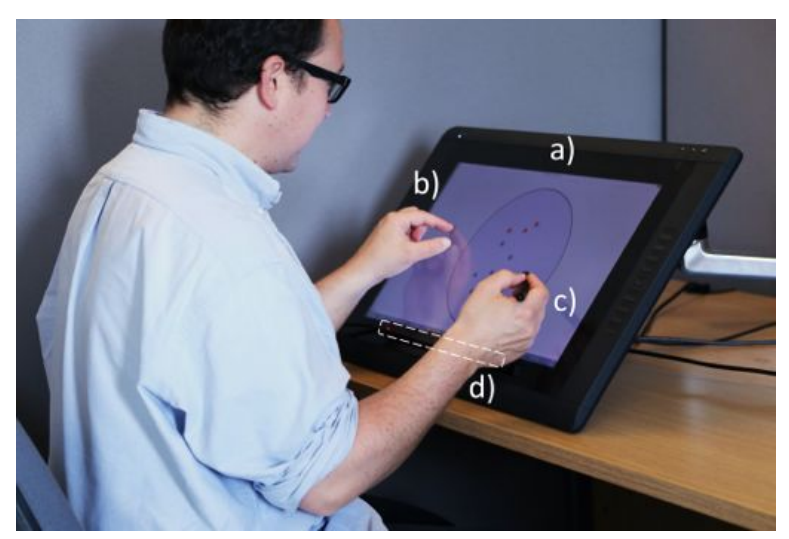

Figure 7: System setup: pen and touch display (a), user's multi-touch input (b), pen input (c), eye tracker (d).

\section{Statistical Analysis}

For the quantitative data, a two-factor repeated-measures ANOVA (Greenhouse-Geisser corrected if sphericity violated) was employed, followed by post-hoc pairwise comparisons with Bonferroni corrections. Qualitative data was analysed with a Friedman test and post-hoc Wilcoxon Signed Rank tests with Bonferroni corrections.

\section{RESULTS}

We report the results based on the initial research questions. Mean values are reported within each bar in the bar diagrams.

\section{Task Completion Time}

For task completion time measures, in the sequence task timing starts when users first touch down and ends when users lift the pen after encircling three targets. For the parallel task, timing starts when users pressed the pen at the line start point, and ends when users lift the pen at the line end point.

The results are presented in Figure 8a. They indicate that the users performed comparatively across the techniques. In the sequence task, technique had a significant effect on task completion time $\left(\mathrm{F}_{2,34}=5.5, \mathrm{p}=.008\right)$. Users performed significantly faster with direct-touch than with pen-touch $(\mathrm{p}=.015)$, no significant differences were found among the remaining comparisons. In the parallel task, technique did not significantly affect task completion time $\left(\mathrm{F}_{2,34}=1, \mathrm{p}=.36\right)$. Factor distance significantly affected performance in the sequence $\left(\mathrm{F}_{1.3,22.9}=173.9, \mathrm{p}<.001\right)$ and parallel task $\left(\mathrm{F}_{1.4,24.8}=110.2\right.$, $\mathrm{p}<.001$, all pairs $\mathrm{p}<.001$ ), though no significant interaction effects between technique and distance were found; neither were any learning effects found across blocks.

\section{Accuracy}

Zoom-accuracy is how accurate users zoomed during pinchto-zoom gestures, i.e. the disparity in centimeter between the position users zoom at and the actual target users should zoom at. We only consider zoom-in gestures, as for zoom-out the target did not matter in our task. We measure zoom-accuracy in each frame during zoom-in gestures. These measures were averaged in each trial; providing the same data base as with task completion time.

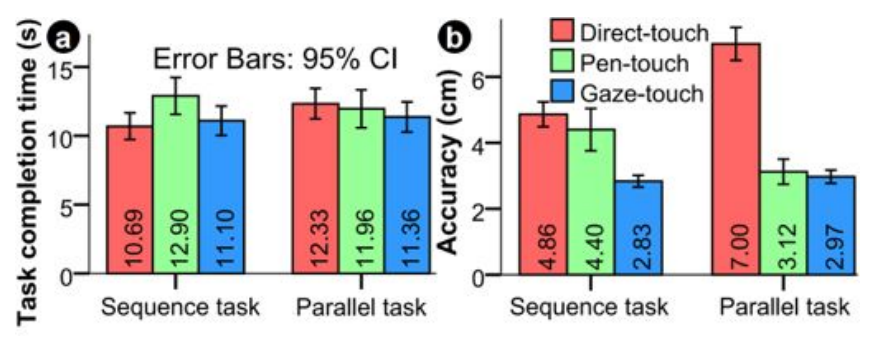

Figure 8: Users performed comparatively in time, and were more accurate with the indirect techniques.

In both tasks, users were most accurate with gaze-touch, then pen-touch, and lastly direct-touch (Figure $8 \mathrm{~b}$ ). This can be accounted to the Dynamic Targeting feature included in both indirect techniques. We found a significant effect of technique on accuracy for the sequence $\left(\mathrm{F}_{2,34}=12.6, \mathrm{p}<.001\right)$ and parallel task $\left(\mathrm{F}_{2,34}=65.4, \mathrm{p}<.001\right)$. In the sequence task, users were more accurate with gaze-touch than direct-touch $(\mathrm{p}<.001)$. Also users were more accurate with gaze-touch than pentouch $(\mathrm{p}=.0021)$. No significant difference was found between direct-touch and pen-touch $(\mathrm{p}=.813)$. In the parallel task, users were more accurate with both gaze-touch and pentouch than direct-touch (both pairs $\mathrm{p}<.001$ ), but no difference was found between pen-touch and gaze-touch $(\mathrm{p}=1.967)$. Further, no learning effects were found across blocks.

We plotted zooming-accuracy during gestures to see how the Dynamic Targeting feature of indirect techniques behaves over time. For this, we collected the average zoomingaccuracy for each frame $(120 \mathrm{hz})$, for each zoom-in gesture that users have performed. This results in a list of gestures where each gesture consists of one accuracy value for each frame. We calculated the time for each frame and plotted as presented in Figure 8. Each gesture begins at Time=0, but the ending time of a gesture is individual for each gesture (see exact durations in Fig. 10a), and we plotted for 1 second. We show the Error Bars (95\%) for each to indicate when the data is becoming too 'spread'.

The zooming-accuracy over time shows that the indirect techniques have stable accuracy over time. In contrast, with direct-touch the accuracy decreases with increasing time. We think this is because first, when users want to zoom exactly on a target, the target will continuously offset away from the touch positions and become more inaccurate over time. Second, there are cases where users deliberately touch offset from the target, so that it is still visible, which yields a continuous inaccuracy during zoom.

\section{Gesture Characteristics}

We now present results on the different gesture characteristics across the used techniques. For this, we conducted a post-hoc analysis on zoom-in gestures. Priorly, we classify zoom-out, zoom-in, and drag gestures based on Avery et al's parameters [2]. We use a minimum factor of 5px movement to classify motion as a gesture. Zoom and drag are distinguished by single and two touch. Zoom is further distinguished to zoomin/-out by checking initial and ending scale of the gesture. 


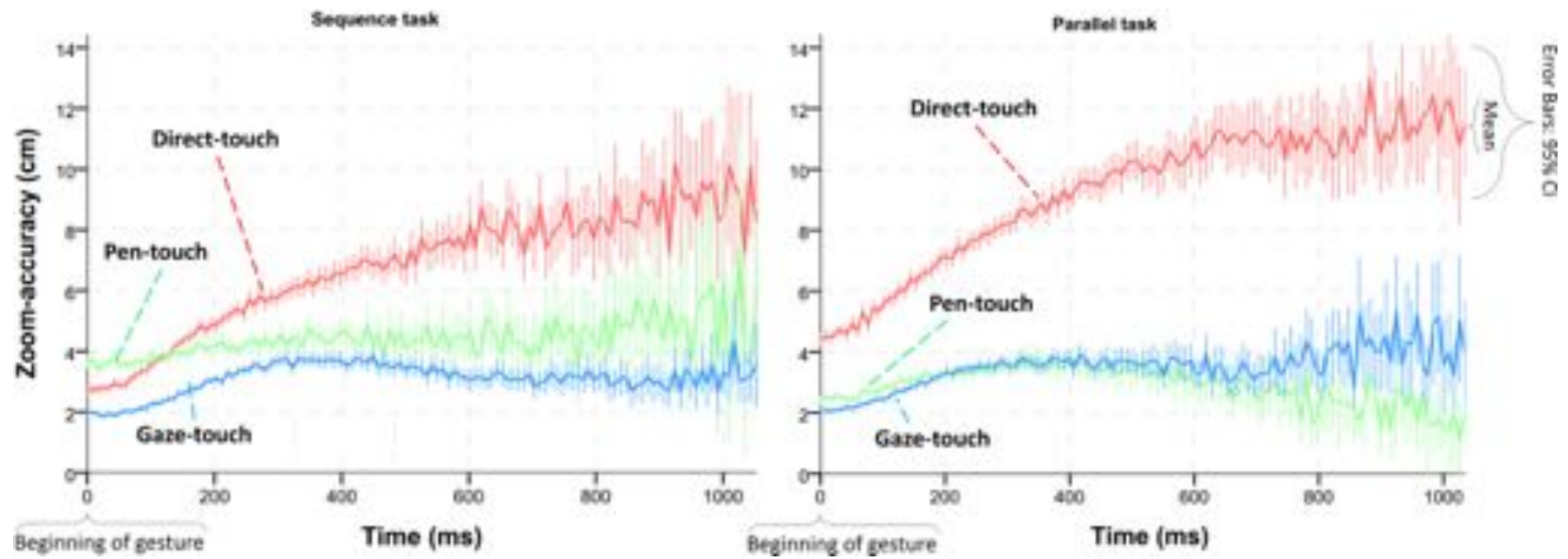

Figure 9: Zoom-accuracy over time during zoom-in gestures, revealing that indirect techniques (pen-touch, gaze-touch) have constant accuracy during zooming, but direct-touch's accuracy decreases over time.

We measured average gesture count and time for each condition. We conducted an ANOVA to see how they are affected by technique, leading to the following findings:

Users performed shorter zoom-in gestures with indirect techniques in the parallel task (Figure 10a): In the parallel task, a significant effect of zoom duration on technique $\left(\mathrm{F}_{1.5,25.2}=18.1, \mathrm{p}<.001\right)$ showed that users performed shorter zoom-in gestures with gaze-touch $(\mathrm{p}<.001)$ and pen-touch $(\mathrm{p}=.0095)$ than with direct-touch. No significant differences among conditions were found for the sequence task.

Users performed less zoom-in gestures with pen-touch than direct-touch in the sequence task (Figure 10b): a significant effect of zoom duration on technique was found for the sequence $\left(\mathrm{F}_{2,34}=7.3, \mathrm{p}=.002\right)$ and the parallel $\left(\mathrm{F}_{2,34}=4.5\right.$, $\mathrm{p}=.018)$. In the sequence task, users performed less zooms with pen-touch than with direct-touch $(\mathrm{p}=.006)$, while other pairs did not yield significant differences.

These findings correlate with our observations, we often saw users performing indirect-touch gestures faster and therefore more frequent than with direct-touch. We think this can be accounted to the indirection: users do not physically see their fingers touching the surface, and therefore just 'quickly' perform a gesture with as least effort as possible. In contrast, with direct-touch users see how their fingers physically touch
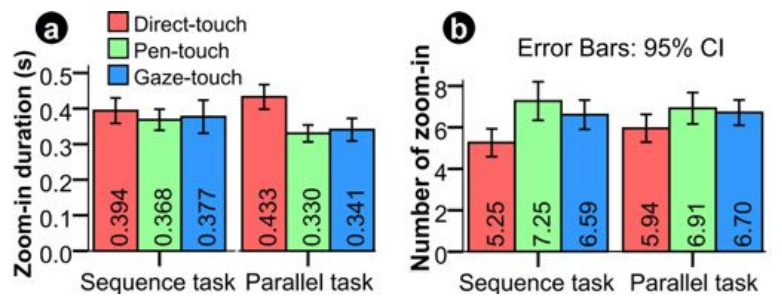

Figure 10: Zoom-in gesture duration and count, indicating different zoom behaviour between direct-touch and the indirect techniques. the screen, making users utilise the pinch gesture with more extensive scales and for longer time.

\section{Parallelism between Pen and Touch}

Parallelism in bimanual input can improve efficiency (as in our parallel task) but also introduce overhead (e.g. when requiring parallelism in a sequence task) $[7,16]$. We now investigate the techniques with regards to their amount of exhibited parallelism between pen and touch modalities. Simultaneous input of both modalities is measured as parallel (i.e. when both pen and touch are in 'drag' mode). We did not consider metrics that include efficiency of parallelism [3], as our study is based on asymmetric bimanual interaction. It is likely that any movement, whether parallel or not, is intended to successfully finish the task. The particular metric we measure when both pen and touch perform a specific number of $\mathrm{cm}$ movement, is the smaller value of both. We computed this value for each condition, and report it as the number of centimeters users would perform in parallel per second.

Figure 11a presents the parallelism results. A significant effect on technique $\left(\mathrm{F}_{1,18}=42.3, \mathrm{P}<.001\right)$ showed an expected result for the sequence task: users performed significantly more parallel movement with pen-touch than with other techniques, as it required bimanual input for zooming (both $\mathrm{p}<.001)$. No difference was found between direct-touch and gaze-touch $(\mathrm{p}=1.49)$. In the parallel task, all techniques showed high parallelism as expected by the design of the task. However, we found a significant effect of technique $\left(\mathrm{F}_{2,34}=5.2, \mathrm{p}=.011\right)$, showing that gaze-touch had less parallelism than pen-touch $(\mathrm{p}=.033)$. While it is clear why pentouch has higher parallelism by design, it is unclear why direct-touch has higher parallelism than gaze-touch in the parallel task.

We plotted parallelism over the duration of each trial to gain further insight into the differences between the techniques. As each trial has a different duration, we normalised the task time between 0 and $100 \%$. For this, we divided the task into 

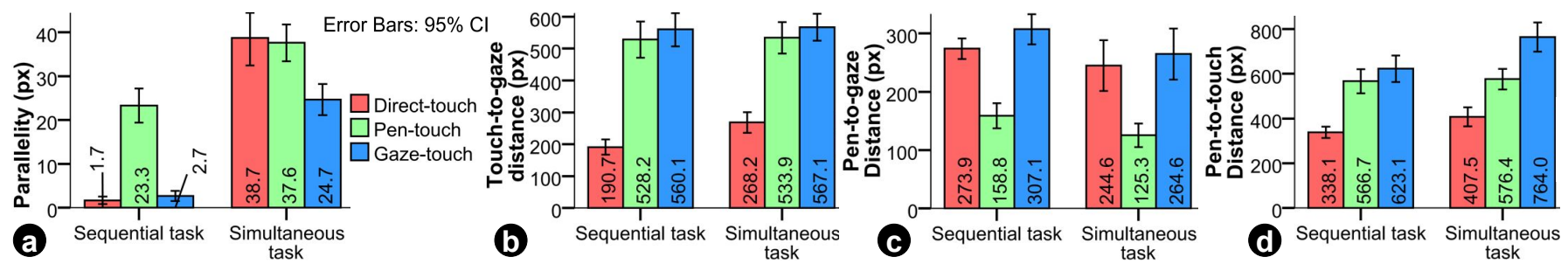

Figure 11: How parallel users performed with the pen and touch modalities (a), and how distant users kept each modality-pair (b, c, d).

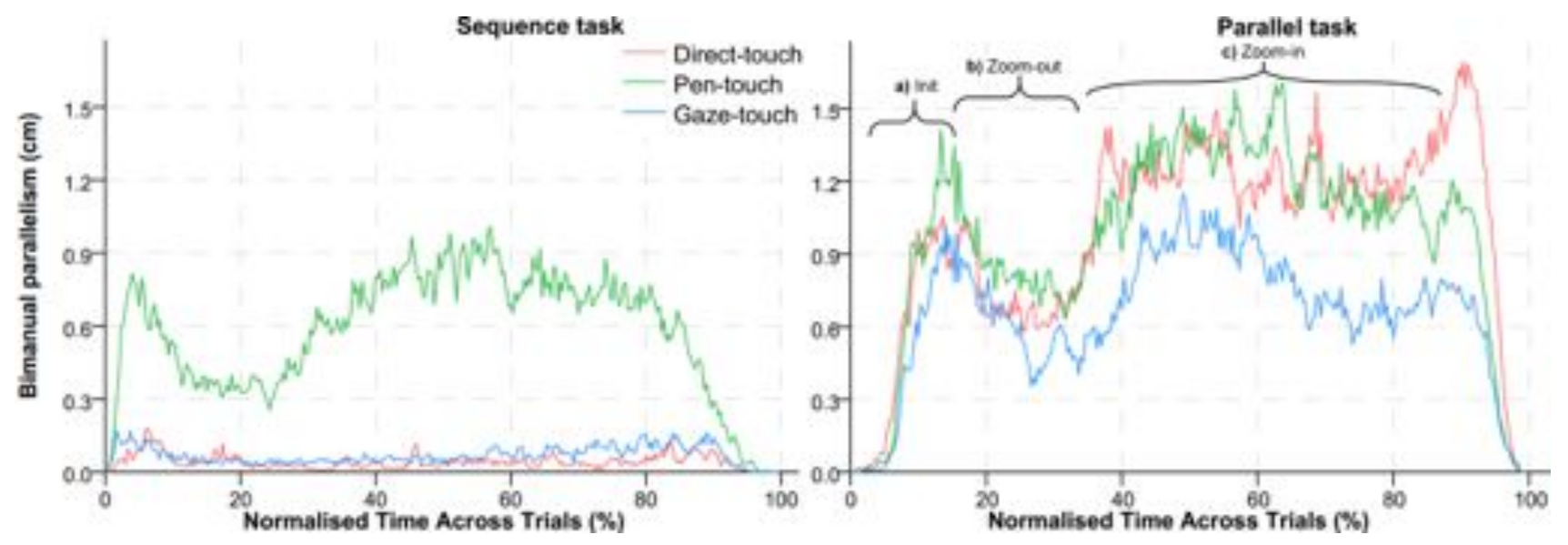

Figure 12: Parallelism of the pen and touch modalities over the duration of each trial. Parallelism denotes the minimum movement in cm that both modalities share. Trial durations are normalised to $0-100 \%$ of time.

300 time segments, and then computed the average parallelism for each time segment, and then distributed the average 300 segments into $100 \%$.

Figure 12 shows the parallelism over time. In the sequence task, only pen-touch has high parallelism, as expected because of the bimanual technique design. In the parallel task, all techniques have parallelism corresponding to the task, with three periods: a peak on parallelism at the start when users initiate pen and touch (Fig. 12a), a period of low parallelism when users zoom-out as no pen movement is needed (b), and then a period of high parallelism when zooming in (c). The difference of parallelism between direct-touch and gaze-touch can be further reduced to the zoom-in period. In light of this, we hypothesise the difference exists because of the interference problem: users often wanted to zoom where the pen was located. This required direct-touch users to move the pen away in order to make space for touch input, in turn increasing parallelism.

\section{Spatial Distribution of Input Modalities}

With direct-touch input it is likely that users look where they touch - but do users return to these patterns with the indirect techniques? To get insights into this we look at how users spatially aligned the pen, touch, and gaze modalities. For this, we analyse the relative distances that users employed during the use of the input modalities. For each trial, we measured the average position of gaze, pen (at both hover/pressed), and touch (zoom-in only, using center of touches). Then we computed the relative distances between the modality positions, for each test condition. Our statistical analysis yielded the following findings (2D heatmap visualisations in Fig. 13):

Users kept pen and touch further apart with the indirect techniques (Figure 11b): Technique significantly affected pen-to-touch distance in the sequence $\left(\mathrm{F}_{2,34}=18.9, \mathrm{p}<.001\right)$ and the parallel task $\left(\mathrm{F}_{2,34}=23.8, \mathrm{p}<.001\right)$. In both tasks, users showed a significantly larger pen-to-touch distance with both gaze-touch and pen-touch compared to direct-touch ( pair $_{\text {pen-touch/direct-touch }} \mathrm{p}=.018$, else pairs $\mathrm{p}<.001$ ). In addition, in the sequence task users kept pen and touch further apart with gaze-touch than pen-touch $(\mathrm{p}=.0181)$. Overall, this indicates that users utilise the screen space for a relaxed touch placement with the indirect techniques.

Users look closer to the pen with pen-touch (Figure 11c): Technique significantly affected pen-to-gaze distance in the sequence $\left(\mathrm{F}_{2,34}=33.2, \mathrm{p}<.001\right)$ and the parallel task $\left(\mathrm{F}_{2,34}=9\right.$, $\mathrm{p}<.001)$. Across both tasks, pen-touch users looked closer to the pen than with the other techniques $(\mathrm{p}<.05)$ (sequence task both pairs $\mathrm{p}<.001$; parallel task pair $_{\text {pen-touch/direct-touch }}$ $\mathrm{p}=.021$, pair ${ }_{\text {pen-touch/gaze-touch }} \mathrm{p}=.009$ ). No significant difference was found between direct-touch and gaze-touch (sequence task $\mathrm{p}=.502$; parallel task $\mathrm{p}=3$ ). This indicates a distinct behaviour of visual attention and pen usage between pen-touch and the other techniques.

Users look closer to their touch positions with direct-touch (Figure 11d): Technique significantly affected touch-to-gaze distance in the sequence $\left(\mathrm{F}_{2,34}=54.2, \mathrm{p}<.001\right)$ and the parallel task $\left(\mathrm{F}_{2,34}=26.7, \mathrm{p}<.001\right)$. Across both tasks, we found that 


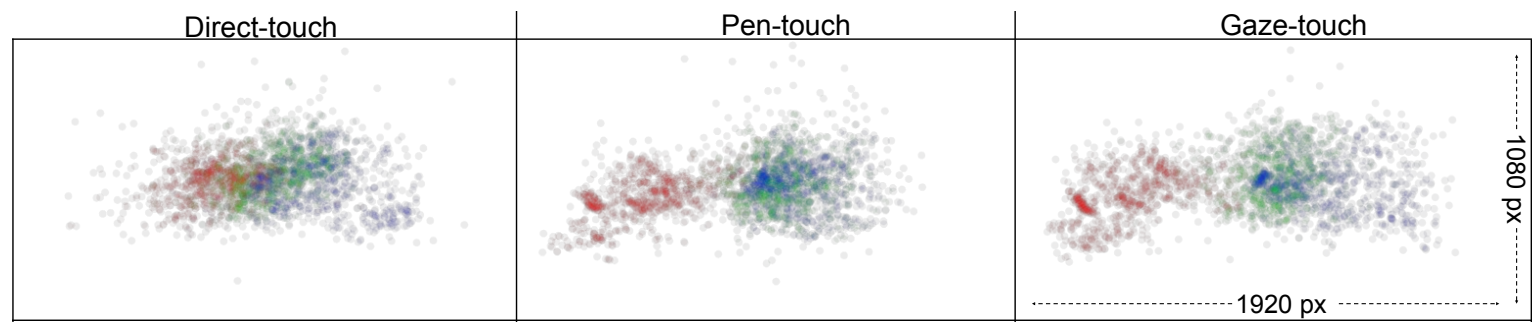

Figure 13: Heatmaps of touch (red), pen (blue), and gaze (green) positions across the screen.

direct-touch users looked significantly closer to their touch positions (all pairs $\mathrm{p}<.001$ ). No significant difference was found between pen-touch and gaze-touch (both pairs $\mathrm{p}=3$ ). Thus users do indeed deviate from the direct-touch pattern, and use indirect-touch offset from their visual focus.

\section{User Feedback}

After each task $\times$ technique block, users filled out a Likert Scale questionnaire. The results are presented in Figure 14.

In the sequence task, a Friedman test found significant differences between conditions for responses on ease $\left(\chi^{2}(2)=7\right.$, $\mathrm{p}=.03)$ and learnability $\left(\chi^{2}(2)=9.8, \mathrm{p}=.007\right)$. Users perceived direct-touch as easier to use $(\mathrm{Z}=-2.49, \mathrm{p}=.039)$ and also as easier to learn $(\mathrm{Z}=-2.65, \mathrm{p}=.024)$ than pen-touch. This was expected as users have significant prior experience with direct-touch.

In the parallel task, significant differences between conditions were found for responses on ease $\left(\chi^{2}(2)=11.5, \mathrm{p}=.003\right)$. First, users found pen-touch easier than direct-touch $(\mathrm{Z}=-$ $2.84, \mathrm{p}=.015)$. In addition, users found gaze-touch easier to use than direct-touch for this task $(Z=-2.49, p=.015)$.

Results of the rankings at the end of the experiment are presented in Figure 15, indicating more preference for gazetouch, and least preference for pen-touch interaction.

Users filled out a questionnaire at the end of the study about why they chose their ranking. The results are mixed across the users. Three users stated that direct-touch is intuitive and familiar ('I don't perceive any difficulty in the task due to the familiarity'), while two users stated that this technique is too much physical effort over time ('it requires too much physical effort and hand coordination').

One user disliked that pen-touch occludes ('the pen was distracting my field of view'), and two users that it is tiring to use ('it is tiring to have to use both hands at once'), while three users were positive about the technique for precision ('my arm movement allowed more precision when zooming into a target circle') and little effort.

Three users disliked gaze-touch as 'it tires the eyes on a gradual basis', 'Hand operation is more intuitive than gaze', and as one user 'found it frustrating at times when the grey circle disappeared off screen', yet six users favoured gaze-touch because of less physical effort ('I did not have to consciously use both hands, which was physically demanding sometimes'), and for easier zooming ('I didn't have to think much about where to zoom as I was usually already looking there').

\section{DISCUSSION}

We discuss the results based on our initial research questions:

Task Completion Time: The times indicate comparable performance across the techniques and tasks; however, pentouch was significantly slower than direct-touch for the sequential task. No differences were found with gaze-touch compared to other techniques. We attribute the lower performance of pen-touch to its necessity of bimanual input. Using two hands for a task that can be accomplished with one hand introduced additional effort. This is partly supported by the qualitative data, where users found pen-touch more difficult than direct-touch in this task.

Accuracy: Across both tasks, users were most accurate with gaze-touch, and least accurate with direct-touch. We found that direct-touch accuracy degrades over time, while the indirect techniques remained at a stable accuracy. This is clearly accounted to the Dynamic Targeting aspect, and is the main benefit of indirect-touch that we found in our experiment. With increasing accuracy of eye trackers, we see potential of more substantial accuracy improvements, as our post-study accuracy tests showed a gaze inaccuracy of approximately $63 \mathrm{px}\left(\approx 1.5^{\circ}\right.$ visual angle $)$. While pen-touch similarly showed this accuracy, the necessity for bimanual input showed a decrease in accuracy in the sequential task, making gaze-touch more suitable for these tasks.

Gesture Characteristics: Users perform zoom gestures slightly different when indirect: they can be shorter in duration (parallel task) and less frequent (sequence task). Of course, a shorter duration of gestures naturally increases the frequency of gestures, and it is unclear whether this is a benefit or drawback. It is possible that users performed them faster to minimise finger movement effort. However, in light that there was no best performer in task completion time, further studies are needed to clarify direct/indirect differences and how they affect performance.

Parallelism between Pen and Touch: Pen-touch has high parallelism as it requires both modalities, which has potential to introduce overhead when using pen and touch in sequence, but which is acceptable in a simultaneous pen and touch task. Gaze-touch showed less parallelism in the simultaneous task, probably as sub-tasks that are normally associated to two hands, are now offloaded to the gaze modality without introducing significant overhead (qualitative ratings did not show increase in eye fatigue). A decrease of paral- 

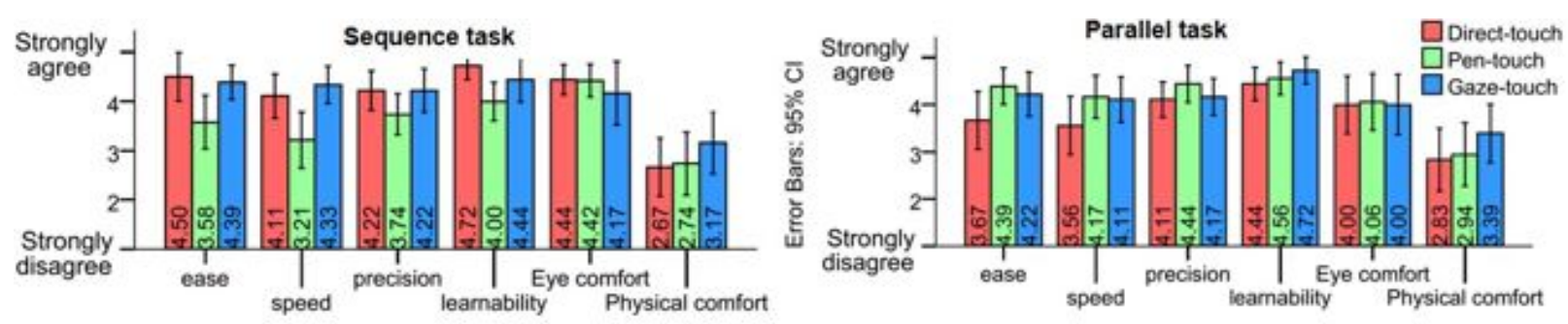

Figure 14: Mean Likert Scale responses from participants

lelism can indicate a release of manual pen and touch effort, yet further long-term studies are needed to clarify this aspect.

Spatial Distribution of Input Modalities: Indirect techniques lead to using pen and touch far apart from each other. We also found that with the pen-touch technique, users look closely at the pen device, and with direct-touch, users look closest to their touch input. These are findings that we expected by the design of the tasks. Interestingly, users held pen and touch further apart during gaze-touch use, thus the addition of gaze decouples both modalities more than other techniques. This can be a problem as users see less physical feedback of their hands but also a benefit as it indicates less occlusion / interference occurrences.

User Feedback: While user ratings slightly correlated with the performance data (direct-touch easier in sequential use, but more difficult in parallel use), the commented feedback was mixed. For each technique, some users found it physically demanding while others found it easy, though the overall ranking indicated preference toward gaze-touch.

In summary, our study pointed to new designs for pan and zoom navigation techniques enabled by the partial indirection of bimanual input. This concept avoids the interference issue of the hands, making simultaneous pen and touch (i.e. when the user wants to issue pen and touch in the same area) easier to use. This can lead to new applications where users e.g. easily navigate the canvas while precisely drawing with the pen; applications that particularly exploit the simultaneity of two modalities while also indicating potential improvements of bimanual UIs in general.

When looking beyond our investigated pan and zoom tasks, interaction designers need to consider limitations such as ambiguous or inaccurate object selection with eye gaze. The gaze-touch technique can be adjusted to only select at touch down, a method that avoids ambiguity and enables new multitouch gesture possibilities [20]. To improve accuracy and thus interaction with small targets, additional precision mechanism can be integrated [24, 34]. Another challenge is to in-

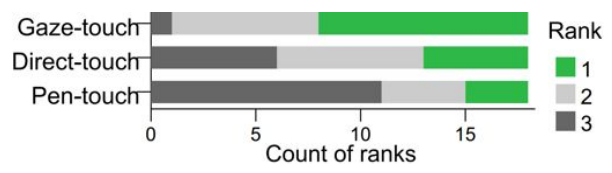

Figure 15: User rankings after the tasks indicate a preference toward gaze-touch. tegrate these techniques into current applications (e.g. Adobe Photoshop). The new techniques can be implemented as new modes in the existing menus; or use more dynamic context switching mechanisms that leverage the user's visual attention during manual input [21].

Integration of gaze to enable indirect-touch raises more questions about the role of gaze. The distinguishing factor for the indirection is the target of manipulation, which is the gaze target, and which is offset from the touch input position. In here we see similarities to input with direct-touch. For instance, with direct pinch-to-zoom it is unlikely to look at the touch positions because they occlude that part of the UI. We often observed users perform zoom-in offset from the actual target, to see the details appearing while zooming. Our quantitative data aligns with this observation, as touches were approximately 4 to $7 \mathrm{~cm}$ (Fig. 11d) offset from the gaze position. Then, the characteristics of direct-touch are similar to gaze-touch: the actual target the user wants to manipulate (and sees) is offset from the touch input position. This would suggest to consider gaze-touch as an extended version of direct-touch, where users control any point they see but can manipulate their point of interest more accurately instead of always being slightly offset from what they want.

\section{CONCLUSION}

In this paper, we explored indirect techniques integrated in a bimanual pen and touch interaction context. For this purpose, we described two configurations where direct pen input is combined with indirect-touch variations: (1) touches redirect to the user's gaze, and (2) touches redirect to the pen's position. These techniques and a control condition (direct pen and touch) were evaluated in a bimanual pen and touch experiment. The results provide new insights into direct versus indirect inputs, how integration indirect-touch retains the dynamics of bimanual pen and touch, and how the gaze modality seamlessly integrates into these contexts.

\section{ACKNOWLEDGEMENTS}

This research was supported by a Google Faculty Research Award ("Gaze-Touch: Multimodal Gaze-and-Touch Input on Interactive Surfaces”).

\section{REFERENCES}

1. Martha Abednego, Joong-Ho Lee, Won Moon, and Ji-Hyung Park. 2009. I-Grabber: Expanding Physical Reach in a Large-display Tabletop Environment Through the Use of a Virtual Grabber. In Proceedings of 
the ACM International Conference on Interactive Tabletops and Surfaces (ITS '09). ACM, New York, NY, USA, 61-64. DOI :

http://dx.doi.org/10.1145/1731903.1731917

2. Jeff Avery, Mark Choi, Daniel Vogel, and Edward Lank. 2014. Pinch-to-zoom-plus: An Enhanced Pinch-to-zoom That Reduces Clutching and Panning. In UIST '14. ACM, 595-604.

3. Ravin Balakrishnan and Ken Hinckley. 2000. Symmetric Bimanual Interaction. In Proceedings of the SIGCHI Conference on Human Factors in Computing Systems (CHI 'O0). ACM, New York, NY, USA, 33-40. DOI : http://dx.doi.org/10.1145/332040.332404

4. Hrvoje Benko, Andrew D. Wilson, and Patrick Baudisch. 2006. Precise Selection Techniques for Multi-touch Screens. In Proceedings of the SIGCHI Conference on Human Factors in Computing Systems (CHI '06). ACM, New York, NY, USA, 1263-1272. DOI : http://dx.doi.org/10.1145/1124772.1124963

5. Anastasia Bezerianos and Ravin Balakrishnan. 2005. The Vacuum: Facilitating the Manipulation of Distant Objects. In Proceedings of the SIGCHI Conference on Human Factors in Computing Systems (CHI '05). ACM, New York, NY, USA, 361-370. DOI :

http://dx.doi.org/10.1145/1054972.1055023

6. Peter Brandl, Clifton Forlines, Daniel Wigdor, Michael Haller, and Chia Shen. 2008. Combining and Measuring the Benefits of Bimanual Pen and Direct-touch Interaction on Horizontal Interfaces. In Proceedings of the Working Conference on Advanced Visual Interfaces (AVI '08). ACM, New York, NY, USA, 154-161. DOI : http://dx.doi.org/10.1145/1385569.1385595

7. W. Buxton and B. Myers. 1986. A Study in Two-handed Input. In Proceedings of the SIGCHI Conference on Human Factors in Computing Systems (CHI '86). ACM, New York, NY, USA, 321-326. DOI :

http://dx.doi.org/10.1145/22627.22390

8. Yves Guiard. 1987. Asymmetric Division of Labor in Human Skilled Bimanual Action: The Kinematic Chain as a Model. In Journal of Motor Behavior, Vol. 19. 486-517.

9. Yves Guiard and Michel Beaudouin-Lafon. 2004. Target Acquisition in Multiscale Electronic Worlds. Int. J. Hum.-Comput. Stud. 61, 6 (Dec. 2004), 875-905.

10. Ken Hinckley, Xiaojun Bi, Michel Pahud, and Bill Buxton. 2012. Informal Information Gathering Techniques for Active Reading. In CHI '12. ACM, 1893-1896.

11. Ken Hinckley, Michel Pahud, Hrvoje Benko, Pourang Irani, François Guimbretière, Marcel Gavriliu, Xiang 'Anthony' Chen, Fabrice Matulic, William Buxton, and Andrew Wilson. 2014. Sensing Techniques for Tablet+Stylus Interaction. In Proceedings of the 27th Annual ACM Symposium on User Interface Software and Technology (UIST '14). ACM, New York, NY, USA, 605-614. DOI :

http://dx.doi.org/10.1145/2642918.2647379

12. Ken Hinckley, Koji Yatani, Michel Pahud, Nicole Coddington, Jenny Rodenhouse, Andy Wilson, Hrvoje Benko, and Bill Buxton. 2010. Pen + Touch $=$ New Tools. In UIST '10. ACM, 27-36.

13. Kenrick Kin, Maneesh Agrawala, and Tony DeRose. 2009. Determining the Benefits of Direct-touch, Bimanual, and Multifinger Input on a Multitouch Workstation. In Proceedings of Graphics Interface 2009 (GI '09). Canadian Information Processing Society, Toronto, Ont., Canada, Canada, 119-124. http: //dl.acm.org/citation. cfm?id=1555880.1555910

14. Celine Latulipe, Craig S. Kaplan, and Charles L. A. Clarke. 2005. Bimanual and Unimanual Image Alignment: An Evaluation of Mouse-based Techniques. In Proceedings of the 18th Annual ACM Symposium on User Interface Software and Technology (UIST '05). ACM, New York, NY, USA, 123-131. DOI : http: //dx.doi.org/10.1145/1095034.1095057

15. Celine Latulipe, Stephen Mann, Craig S. Kaplan, and Charlie L. A. Clarke. 2006. symSpline: Symmetric Two-handed Spline Manipulation. In Proceedings of the SIGCHI Conference on Human Factors in Computing Systems (CHI '06). ACM, New York, NY, USA, 349-358. DOI : http: //dx.doi.org/10.1145/1124772.1124825

16. Andrea Leganchuk, Shumin Zhai, and William Buxton. 1998. Manual and Cognitive Benefits of Two-handed Input: An Experimental Study. ACM Trans. Comput.-Hum. Interact. 5, 4 (Dec. 1998), 326-359. DOI : http://dx.doi.org/10.1145/300520.300522

17. Pedro Lopes, Daniel Mendes, Bruno Araújo, and Joaquim A. Jorge. 2011. Combining Bimanual Manipulation and Pen-based Input for 3D Modelling. In Proceedings of the Eighth Eurographics Symposium on Sketch-Based Interfaces and Modeling (SBIM '11). ACM, 15-22.

18. Fabrice Matulic and Moira Norrie. 2012. Empirical Evaluation of Uni- and Bimodal Pen and Touch Interaction Properties on Digital Tabletops. In ITS ' 12. ACM, 143-152.

19. Mathieu Nancel, Julie Wagner, Emmanuel Pietriga, Olivier Chapuis, and Wendy Mackay. 2011. Mid-air Pan-and-zoom on Wall-sized Displays. In Proceedings of the SIGCHI Conference on Human Factors in Computing Systems (CHI '11). ACM, New York, NY, USA, 177-186. DOI : http://dx.doi.org/10.1145/1978942.1978969

20. Ken Pfeuffer, Jason Alexander, Ming Ki Chong, and Hans Gellersen. 2014. Gaze-touch: Combining Gaze with Multi-touch for Interaction on the Same Surface. In Proceedings of the 27th Annual ACM Symposium on User Interface Software and Technology (UIST '14). 
ACM, New York, NY, USA, 509-518. DOI :

http: //dx.doi.org/10.1145/2642918.2647397

21. Ken Pfeuffer, Jason Alexander, Ming Ki Chong, Yanxia Zhang, and Hans Gellersen. 2015. Gaze-Shifting:

Direct-Indirect Input with Pen and Touch Modulated by Gaze. In Proceedings of the 28th Annual ACM Symposium on User Interface Software \& Technology (UIST '15). ACM, New York, NY, USA, 373-383.

DOI : http://dx.doi.org/10.1145/2807442.2807460

22. Ken Pfeuffer, Jason Alexander, and Hans Gellersen. 2015. Gaze+touch vs. Touch: Whats the Trade-off When Using Gaze to Extend Touch to Remote Displays?. In Human-Computer Interaction INTERACT 2015 (Lecture Notes in Computer Science), Vol. 9297. Springer International Publishing, 349-367. DOI : http://dx.doi.org/10.1007/978-3-319-22668-2_27

23. Sophie Stellmach and Raimund Dachselt. 2012a. Investigating Gaze-supported Multimodal Pan and Zoom. In Proceedings of the Symposium on Eye Tracking Research and Applications (ETRA '12). ACM, New York, NY, USA, 357-360. DOI : http: //dx.doi.org/10.1145/2168556.2168636

24. Sophie Stellmach and Raimund Dachselt. 2012b. Look \& Touch: Gaze-supported Target Acquisition. In CHI '12. ACM, 2981-2990.

25. Jayson Turner, Jason Alexander, Andreas Bulling, and Hans Gellersen. 2015. Gaze+RST: Integrating Gaze and Multitouch for Remote Rotate-Scale-Translate Tasks. In Proceedings of the 33rd Annual ACM Conference on Human Factors in Computing Systems (CHI '15). ACM, New York, NY, USA, 4179-4188. DOI :

http://dx.doi.org/10.1145/2702123.2702355

26. Simon Voelker, Andrii Matviienko, Johannes Schöning, and Jan Borchers. 2015. Combining Direct and Indirect Touch Input for Interactive Workspaces Using Gaze Input. In Proceedings of the 3rd ACM Symposium on Spatial User Interaction (SUI '15). ACM, New York, NY, USA, 79-88. DOI :

http://dx.doi.org/10.1145/2788940.2788949

27. Daniel Vogel and Ravin Balakrishnan. 2010. Occlusion-aware Interfaces. In Proceedings of the SIGCHI Conference on Human Factors in Computing Systems (CHI'10). ACM, 263-272.

28. Daniel Vogel and Patrick Baudisch. 2007. Shift: A Technique for Operating Pen-based Interfaces Using Touch. In Proceedings of the SIGCHI Conference on Human Factors in Computing Systems (CHI '07). ACM, New York, NY, USA, 657-666. DOI :

http://dx.doi.org/10.1145/1240624.1240727

29. Daniel Wigdor, Hrvoje Benko, John Pella, Jarrod Lombardo, and Sarah Williams. 2011. Rock \&\#38; Rails: Extending Multi-touch Interactions with Shape Gestures to Enable Precise Spatial Manipulations. In Proceedings of the SIGCHI Conference on Human Factors in Computing Systems (CHI '11). ACM, New
York, NY, USA, 1581-1590. DOI :

http://dx.doi.org/10.1145/1978942.1979173

30. Mike Wu, Chia Shen, Kathy Ryall, Clifton Forlines, and Ravin Balakrishnan. 2006. Gesture Registration, Relaxation, and Reuse for Multi-Point Direct-Touch Surfaces. In Proceedings of the First IEEE International Workshop on Horizontal Interactive Human-Computer Systems (TABLETOP '06). IEEE Computer Society, Washington, DC, USA, 185-192. DOI : http://dx.doi.org/10.1109/TABLETOP.2006.19

31. Ka-Ping Yee. 2004. Two-handed Interaction on a Tablet Display. In CHI '04 Extended Abstracts on Human Factors in Computing Systems (CHI EA '04). ACM, New York, NY, USA, 1493-1496. DOI : http://dx.doi.org/10.1145/985921.986098

32. Dongwook Yoon, Nicholas Chen, François Guimbretière, and Abigail Sellen. 2014. RichReview: Blending Ink, Speech, and Gesture to Support Collaborative Document Review. In Proceedings of the 27th Annual ACM Symposium on User Interface Software and Technology (UIST '14). ACM, New York, NY, USA, 481-490. DOI :

http: //dx.doi.org/10.1145/2642918.2647390

33. Robert Zeleznik, Andrew Bragdon, Ferdi Adeputra, and Hsu-Sheng Ko. 2010. Hands-on Math: A Page-based Multi-touch and Pen Desktop for Technical Work and Problem Solving. In UIST '10. ACM, 17-26.

34. Xinyong Zhang, Xiangshi Ren, and Hongbin Zha. 2008. Improving Eye Cursor's Stability for Eye Pointing Tasks. In Proceedings of the SIGCHI Conference on Human Factors in Computing Systems (CHI '08). ACM, New York, NY, USA, 525-534. DOI : http://dx.doi.org/10.1145/1357054.1357139 